Journal of Analytical Chromatography and Spectroscopy (2018) Volume 1 doi:10.24294/jacs.v1i2.626

\title{
Isolation detection and characterization of syringolin a produced from the probiotic strain Bacillus cereus isolated from donkey milk
}

\author{
Rashmi. D ${ }^{1}$, Sharmila.T ${ }^{1}$, Sushama Patil ${ }^{2}$, Onkar Apine ${ }^{2}$, Srinivas Sistla ${ }^{3 *}$, Jyoti Jadhav ${ }^{2}$ \\ ${ }^{1}$ Department of Microbiology and Biotechnology, Bangalore University, Bangalore- 560056 Karnataka, India. \\ ${ }^{2}$ Department of Biotechnology, Shivaji University, Kolhapur 416004 \\ ${ }^{3}$ GE Healthcare Life Sciences, John F Welch Technology Centre, Whitefield Road, Bangalore, 560048, India \\ *Present address: Institute of Structural Biology Drug Discovery and Development, Virginia Commonwealth University \\ Richmond, VA, USA 23219
}

\begin{abstract}
Syringolin A is a non-ribosomal virulence factor secreted by few Pseudomonas strains. Syringolin A is a well known irreversible proteasome inhibitor and antitumor compound. The present study is focused on the extraction of Syringolin A through a non-tedious and economical process. Syringolin A is extracted from culture supernatants by the immiscible organic layer by mixing of dichloromethane or chloroform (trichloromethane). Syringolin A was identified by the characteristic peak at $350 \mathrm{~nm}$ by UV spectra. The compound was further characterized by Thin Layer Chromatography (TLC) with the retention value, $\mathrm{R}_{\mathrm{f}}$ was found to be within the range of $0.78-0.83$ run using a combination of solvent systems water and methanol. The molecular weight of the compound determined as 492.2614 $\mathrm{g} \mathrm{mol}^{-1}$ identified and analyzed by UHPLC-QTOF-MS analysis. Due to its significant pharmacological importance in proliferative diseases, further studies on the production and optimization of these compounds are necessary.

Keywords: Chloroform; Dichloromethane; Thin Layer Chromatography; UHPLC-QTOF-MS; UV-Visible spectrophotometer
\end{abstract}

\section{Introduction}

Plants have evolved an innate immune system as a defense against pathogens. Plant bacterial defense systems were studied in great detail as they have paved the way for the identification of novel molecules of pharmacological importance. The scientific interest for the search of natural means of microbial inhibitors or defense molecules has paved the way for the study on natural antibiotics called bacteriocins and bacteriocin-like inhibitory substances (BLIS) which are produced by a wide range of bacterial species have gained huge attention. BLIS involve a group of non-ribosomally combined peptides that manifests narrow spectrum antimicrobial properties. BLIS can be considered as anti-toxins, they vary from traditional antibiotics as these are proteinaceous in nature. BLIS is rapidly destroyed by the action of proteases and degraded in the human $\operatorname{gut}^{[1]}$.

Syringolin A isolated from supernatants of the Pseudomonas syringae $p v$. Syringae cultures is a peptide virulence factor, synthesized by non-ribosomal peptide systems of these bacterial strains as a defense system. Syringolin A binds irrepressible to proteasome targets and gained significant importance as an anti-proliferative molecule ${ }^{[2]}$.

A novel method for extraction and purification of small bacteriocins (small peptides with antimicrobial properties) were carried out successfully by stirring the cell-free broth with toluene (solvent) to obtain a suspension into the solvent layer. The order of yield by solvent extraction was found to be $90 \%$ in a single step process ${ }^{[3]}$. A new solvent affinity method was developed to concentrate lacidin from the culture of Lactobacillus acidophilus OSU133. This method partitions the bacteriocin at the interface between chloroform and the cell-free culture supernatant. Compared with other extraction procedures like ammonium sulfate precipitation and cell acidification, the new method effectively recovers higher bacteriocin yield and results in relatively clean preparations. Recovery of bacteriocin by the chloroform extraction was $>10$-fold and 100-fold greater, compared with ammonium sulfate precipitation and cell acidification

Copyright (C) 2018 Rashmi. D et al.

doi: 10.24294/jacs.v1i2.626

EnPress Publisher LLC. This work is licensed under the Creative Commons Attribution-NonCommercial 4.0 International License (CC BY-NC 4.0).

http://creativecommons.org/licenses/ by/4.0/ 
methods respectively ${ }^{[4]}$. There are many reports on the characterization of Syringolin A. A bacteriocin isolated from Lactobacillus paracasei HL32 was characterized from culture supernatants by ultraviolet spectrophotometer that showed maximum peak absorbance at $214 \mathrm{~nm}$ and $254 \mathrm{~nm}$ and further thin-layer chromatography was carried out ${ }^{[5]}$.

The antimicrobial compounds in the cell-free fermented medium were also extracted by the methanol-acetone solvent system. The solvent extract was partially purified by thin-layer chromatography which showed one main spot at $\mathrm{R}_{\mathrm{f}}$ 0.8. Antimicrobial activity of the methanol-acetone extract was retained at $\mathrm{pH} 2.5$ to 6.0 but was lost after treating with proteolytic enzymes ${ }^{[6]}$. Bacillus subtilis isolated from Tunisian soil showed strong antimicrobial activity. Cell culture supernatant was subjected to thin layer chromatography that showed the spots with $\mathrm{R}_{\mathrm{f}}$ values of 0.70 and 0.82 which exhibited antibacterial activity ${ }^{[7]}$.

The qualitative and quantitative analysis of antimicrobial bioactive peptides is involved in the proteomic studies. The proteins are analyzed by a peptidomics approach that aims at simultaneous visualization and identification of the whole peptide compound by the use of powerful hybrid mass spectrometric tools such as Quadrupole- Time of Flight Liquid Chromatography-Mass Spectroscopy (Q-TOF LC/MS ${ }^{[8,9]}$. The technique provides a highly useful qualitative and quantitative analysis of complicated components which helps in accurate mass measurement and used to determine the elemental composition of peptides thereby confirming the proposed structures ${ }^{[10]}$.

In the present study, a compound was extracted from the bacterial culture isolated from Donkey milk. This compound was characterized further by UV absorbance, TLC, and HPLC MS to be a Syringolin A.

\section{Experimental part}

\subsection{Cultures and Media}

The isolation bacteria from donkey milk begun with collecting the milk aseptically in a sterile bottle used immediately within 2 hours or stored at $4^{\circ} \mathrm{C}$. Raw and pasteurized milk was used for isolation of bacteria and was serially diluted in the range from $10^{-1}$ to $10^{-5} \mathrm{CFU} / \mathrm{ml}$ in sterile distilled water. Isolation of bacteria was carried out for the dilutions $10^{-3}$ to $10^{-5} \mathrm{CFU} / \mathrm{ml}$ by pour plate method on nutrient agar, incubated at $37^{\circ} \mathrm{C}$ for $24-48 \mathrm{hrs}{ }^{[11]}$. The optimization and growth of the bacterial strains are discussed elsewhere ${ }^{[12]}$.

\subsection{Ultraviolet Spectroscopy}

The cell-free supernatant of the strain Bacillus cereus isolated from Donkey milk was scanned in the range of 220 $-360 \mathrm{~nm}$ using Shimadzu spectrophotometer (Kyoto, Japan) for spectral absorbance measurements for the characteristic of bacteriocin-like peptide bonds ${ }^{[13,14]}$. The media broth was taken as a reference baseline. The controls were also employed and nisin is used as a positive control in all the analytical experiments.

\subsection{Solvent Extraction of the Cell-Free Supernatant}

Solvents like dichloromethane and chloroform were mixed with cell-free supernatant in 7:3 v/v separately based on the miscibility property, shaken vigorously at room temperature for $20 \mathrm{mins}$ to ensure bacteriocin-like compounds were to extracted into the solvent layer and settled for $24 \mathrm{hrs}^{[4,16]}$. The organic layer where required was evaporated by Buchi rotavapor (R-300 Cole Parmer IL, USA)

\subsection{Thin Layer Chromatography (TLC) Profiling for the Extract}

TLC was performed with the extracts from dichloromethane and chloroform which exhibited positive antibacterial activity against the indicator organisms. Each of $10 \mu \mathrm{L}$ extracts was spotted on the pre-coated thin layer silica plates (TLC silica gel $60 \mathrm{Fe}_{254}$ ) that were cut in the size of $7 \times 3 \mathrm{~cm}$. The samples were spotted on plates manually using the capillary tubes. Different combination of the solvent system was tried and optimized the best mobile phase for the extracts under the laboratory conditions of $25 \pm 5{ }^{\circ} \mathrm{C}$ and relative humidity of $40-50 \%$. The TLC chamber was made saturated with the same solvent system used for the elution of the proteins ${ }^{[17,18]}$.

A volume of $10 \mu \mathrm{L}$ of the thick immiscible layer formed between the supernatant and chloroform was taken which served as the test solution. The solvent systems of different polarities were tried to select the solvent system capable of showing better resolution after the elution. The best solvent system selected was chloroform: methanol $(6: 4 \mathrm{v} / \mathrm{v})$ ratio. The separation of proteins was based on the differential partition coefficient of stationary and mobile phase. The developed chromatogram was air dried and observed for the bands under ultraviolet (UV) light at both shortwave (254 
$\mathrm{nm})$ and longwave $(366 \mathrm{~nm})$. The movement of analyte was expressed as partition coefficient, indicated by retention factor $\left(\mathrm{R}_{\mathrm{f}}\right)$ which is calculated by the formula ${ }^{[19]}$.

$$
\mathrm{R}_{\mathrm{f}}=\frac{\text { Distance traveled by the analyte }}{\text { Distance traveled by the solvent front }}
$$

\subsection{Ultra High-Performance Liquid Chromatography-Mass Spectrometry} (UHPLC-QTOF-MS) Analysis

The molecular mass of the compound was determined by Quadrupole-TOF LC-MS using the instrument Agilent Series 1290 infinity rapid resolution LC system interfaced with electrospray ionization (ESI) to an Agilent 6540 UHD Accurate-Mass Q-TOF LC/MS (Agilent Technologies, Palo Alto, CA, USA). Chromatographic separation was achieved using different columns, initially to optimize separation conditions to acquire maximum metabolites. A reverse phase $\mathrm{C}_{18}$ ZORBAX RRHD Eclipse Plus analytical column (1200 bar, $1.8 \mu \mathrm{m}$, pore size $\left.95 \AA, 160 \mathrm{~m}^{2} / \mathrm{g}\right)($ Agilent Technologies, Palo Alto, CA, USA). The injected sample volume was $2 \mu 1$ in positive ionization mode. The mobile phases used were eluent a containing formic acid in water, and eluent B with formic acid in methanol. Gradient elution was carried out as follows: eluent B 5-45\% from 0 to 8 min; eluent B $45-90 \%$ from 8 to $10 \mathrm{~min}$, B $90 \%$ from 10 to 15 min; eluent B 3\% from 15 to $20 \mathrm{~min}$. A constant flow rate was used for elution. To maintain mass accuracy during runtime, a reference mass solution with purine and Hexakis(1H,1H,3H-perfluoropropoxy)phosphazene was used, with $\mathrm{m} / \mathrm{z} 121.0508$ and $\mathrm{m} / \mathrm{z} 922.0097$ reference ions respectively in positive ionization mode.

The mass spectrometer performed different runs in positive ionization mode over a mass range from 100 to 1700 $\mathrm{Da}$ at $4 \mathrm{GHz}$ high-resolution mode with a scan duration of 2 spectra/s in centroid and profile mode. The mass spectrometer parameter settings used for the measurement were as follows: capillary voltage: $4.5 \mathrm{kV}$ (positive ion mode) nebulizer pressure: $35 \mathrm{psi}$, drying gas: $15 \mathrm{~L} / \mathrm{min}$, gas temperature: $30{ }^{\circ} \mathrm{C}$, skimmer voltage $65 \mathrm{~V}$, octapole RF $750 \mathrm{~V}$, and fragmented voltage 150 V. Data was collected using Mass Hunter workstation software (Agilent, version B.05.00). The analysis resulted in the determination of the molecular weight of the compound extracted and purified ${ }^{[20]}$. Identification of compounds was accomplished by comparisons of observed accurate mass and MS/MS spectra with accurate mass data and spectra in the Metlin Metabolomics and Tandem MS Database (Scripps Center for Metabolomics, La Jolla, CA), which is licensed in the Mass Hunter software and is available online (http://metlin.scripps.edu/index.php).

\subsection{Surface Plasmon Resonance (SPR)}

Surface Plasmon Resonance interactions were done on a Biacore T200 instrument (GE Healthcare Life Sciences, Bangalore, India). The whole cells were immobilized onto the CM5 (Carboxy methylated) gold chip using amine coupling chemistry. The dextran matrix on the gold chip was activated with a $1: 1$ mixture of $0.4 \mathrm{M}$ 1-ethyl-3-(3-dimethylaminopropyl) carbodiimide (EDC) and 0.1 M N-hydroxy succinimide (NHS) to create reactive succinimide esters. For immobilization, the free carboxyl groups of the bacterial amine residues were activated with EDC/NHS, then reacted with the ethylene diamine-derivatized carboxymethyl dextran sensor chip to obtain the desired ligand concentrations. The standard activation time required to reach the immobilization level is $7 \mathrm{mins}^{[21]}$. The unreacted esters are blocked with $1 \mathrm{M}$ ethanolamine. The immobilization level is $260 \mathrm{RU}$. SPR measurements were carried out in phosphate buffer saline $(0.1 \mathrm{M}$ phosphate buffer with $27 \mathrm{mM} \mathrm{KCl}$ and $1.37 \mathrm{M} \mathrm{NaCl} 0.005 \%$ polysorbate $20 \mathrm{pH}$ 7.4). Onto the immobilized bacterial cells to which the analyte, i.e., cell-free supernatant to be detected is passed and the binding response at a single concentration is detected in a real time mode. Nisin at the concentration of 1ppm $(1 \mu \mathrm{g} / \mathrm{mL})$ is used as a positive control and nutrient broth as a negative control. Data was collected with the Biacore T200 control software version 3.0. Experiments were performed by monitoring the refractive index changes as a function of time under a constant flow rate of $10 \mu \mathrm{l} / \mathrm{min}$. The relative amount of active compound bound to the indicator 
organism was determined by measuring the net increase in refractive index over time compared to control running buffer. There is an inline subtraction from the reference surface during the run. The result from the detection of the change in RI is displayed as a sensorgram plotted with the binding response (RU) on Y-axis against time in seconds on X-axis.

\section{Results and Discussion}

\subsection{Growth of culture and extraction of Syringolin A}

The new single-step solvent extraction procedure which saves time and is easy to perform is optimized for the production of bacteriocin-like compound. The semi-polar solvents such as dichloromethane and trichloromethane (chloroform) mixture were used to easily separate, purify and characterize the compound. The studies conducted by us paved a way to perform a versatile extraction and recovery method which seemed to be 10 -fold greater extractability than the ammonium sulfate precipitation. Similar methods were reported in the literature based on the solvent affinity of bacteriocins like subtilin, bacillicin, pediocin, and nisin from cultures of Bacillus subtilis ATCC 6633, B. subtilis OSY1115/C, Pediococcus acidilactici PO2 and Lactococcus lactis ATCC 11454, respectively ${ }^{[4]}$.

\subsection{UV-Visible Spectrophotometer}

The crude compounds in cell-free supernatants displayed characteristic protein peaks in the UV spectrums (figure 1) at $210 \mathrm{~nm}$ and $350 \mathrm{~nm}$ for the samples of broth, and Bacillus cereus (DM7) respectively. The diffusible bioactive compounds from Bacillus cereus that exhibited potent antibacterial activity was characterized by subjecting to UV-Visible spectrophotometer. The analysis of antimicrobial peptides by spectroscopic methods is not practiced commonly, but it gives a clear idea of the probable presence of bacteriocins in the cell-free supernatants. The UV-visible spectrum peaks clearly indicated the presence of aromatic amino acids. Teixeira et al. ${ }^{[14]}$ reported the characterization of the antimicrobial peptide produced by Bacillus subtilis by spectrometry.

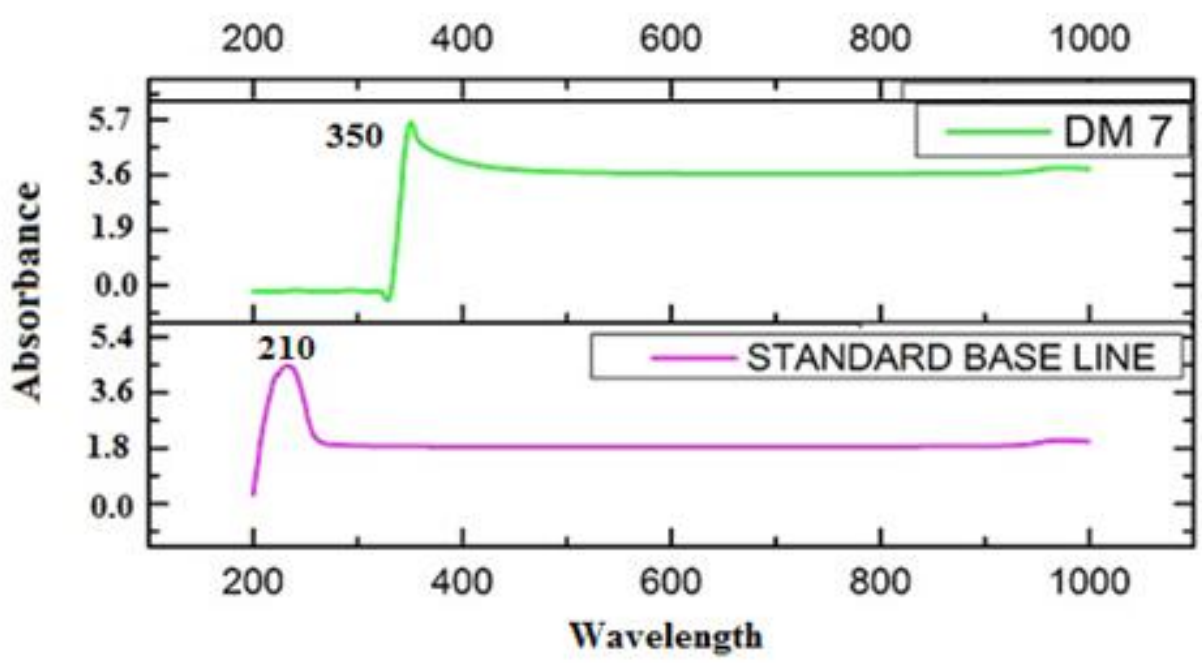

Figure 1; UV-Visible spectrogram of the cell-free supernatant recorded in the range of $200-400 \mathrm{~nm}$ with a $1 \mathrm{~cm}$ path length. Standard baseline- baseline with Broth as reference and DM7- B. cereus culture Supt.

\subsection{Thin layer chromatography (TLC) profiling for the extract of B. cereus (DM7)}

Thin layer chromatogram illustrates the presence of a single prominent fluorescent band observed under UV short wavelength. The chromatogram was developed with chloroform: methanol $(6: 4 \mathrm{v} / \mathrm{v})$ ratio which effectively separated the protein. The retention value, $R_{\mathrm{f}}$ was found to be in the range of $0.78-0.83$ which was spotted in figure 2 . Thin layer chromatographic profiling was carried out for the solvent extracts of $\mathrm{B}$. cereus which rendered an inexpensive and rapid route for the tentative identification of bacteriocin molecules in the extracts. Further, the bands were dissolved in $0.1 \%$ TFA in Acetonitrile solution and subjected to UHPLC-QTOF-MS analysis. 


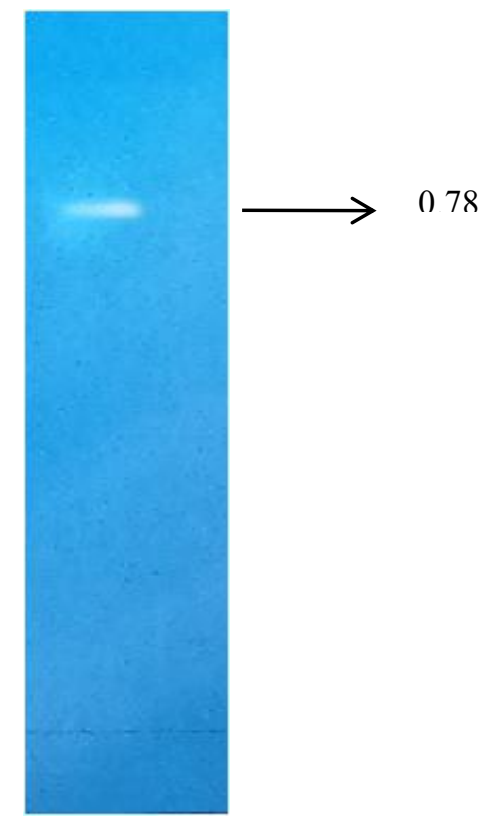

Figure 2; Thin layer chromatograms of the extracts from B. cereus.

\subsection{UHPLC-QTOF-MS analysis of an extract of B. cereus (DM7)}

The UHPLC-QTOF-MS analysis of B. cereus (DM7) revealed the compound as Syringolin A with the retention time of $4.961 \mathrm{~min}$ illustrated in figure 3. Syringolin A ring structure composed of 5-methyl-4-amino-2-hexenoic acid and 3,4-dehydrolysine with the molecular formula $\mathrm{C}_{24} \mathrm{H}_{39} \mathrm{~N}_{5} \mathrm{O}_{6}$ and molecular weight $492.2614 \mathrm{~g} \mathrm{~mol}^{-1}$. The compound exhibited a good mass spectrometric response in the positive ion mode. The mass to charge spectral peak was observed at $492.26 \mathrm{~m} / \mathrm{z}$ and on further MS-MS spectrum provided the fragmentation pattern at $493.26 \mathrm{~m} / \mathrm{z}$ show a characteristic fragment of 381.152 that corresponded to the compound Syringolin A with the accuracy difference of $0.3 \mathrm{ppm}$.
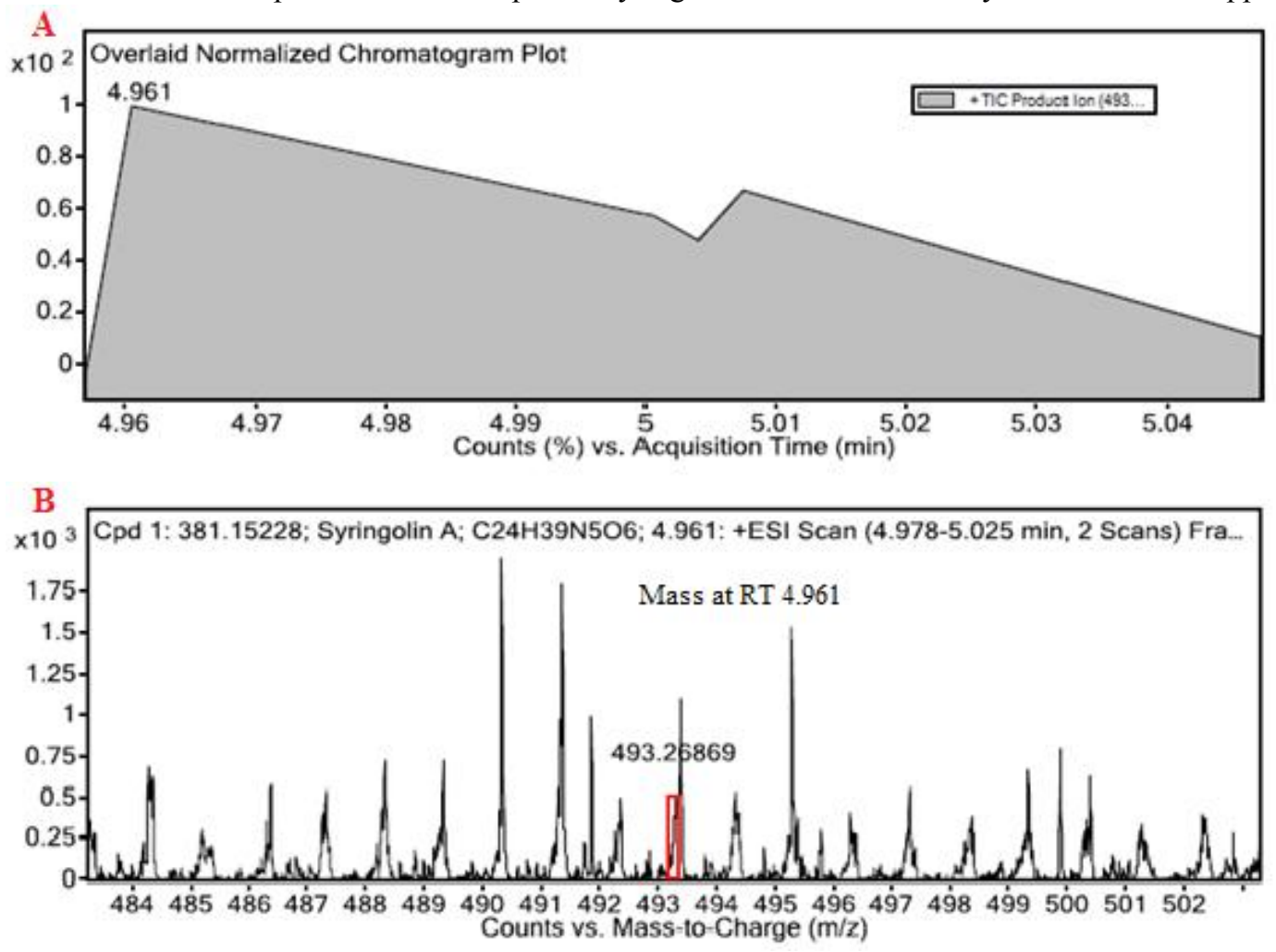

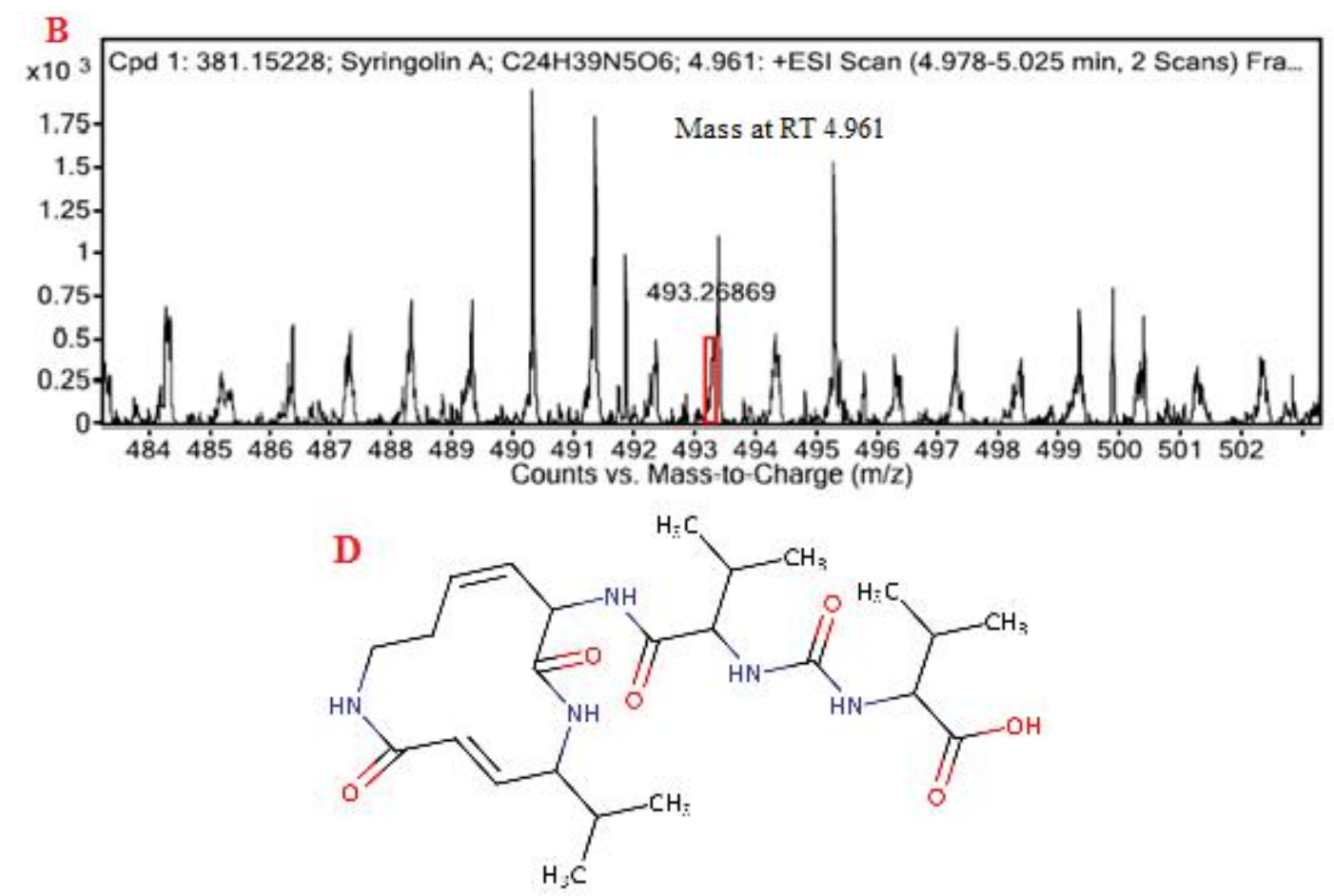

Figure 3; LC/MS spectrum of B.cereus extract. The retention time of the B.cereus extract; B- MS spectra B.cereus extract; CFragmentation pattern of Syringolin A and D-predictive structure of Syringolin A.

The molecular identification of TLC extracts of B.cereus was determined by chromatographic separation and mass spectrometric analysis by Ultra high-performance liquid chromatography coupled to quadrupole time-of-flight mass spectrometry (UHPLC-QTOF-MS). Through the available reports reviewed, the use of UHPLC-QTOF-MS for the bacteriocin identification is for the first time which revealed the compounds present in extracts. The assay provides a wide inspection dynamic range Q-TOF sensitivity which offers versatile, reliable and high accurate mass analysis.

\subsection{Surface Plasmon Resonance}

Biacore was used as an analytical technique to monitor the binding of isolated syringolin A to bacterial cells. The immobilized cells show binding and also the cell culture media shows no binding and can be used as a control. Many samples were screened for the presence of syringolin A and only the isolated Bacillus cultures from Donkey milk shows binding. Figure 4. Shows the binding of single extract from the Bacillus culture to immobilized cells. The binding is slow and gradual and shows no dissociation.

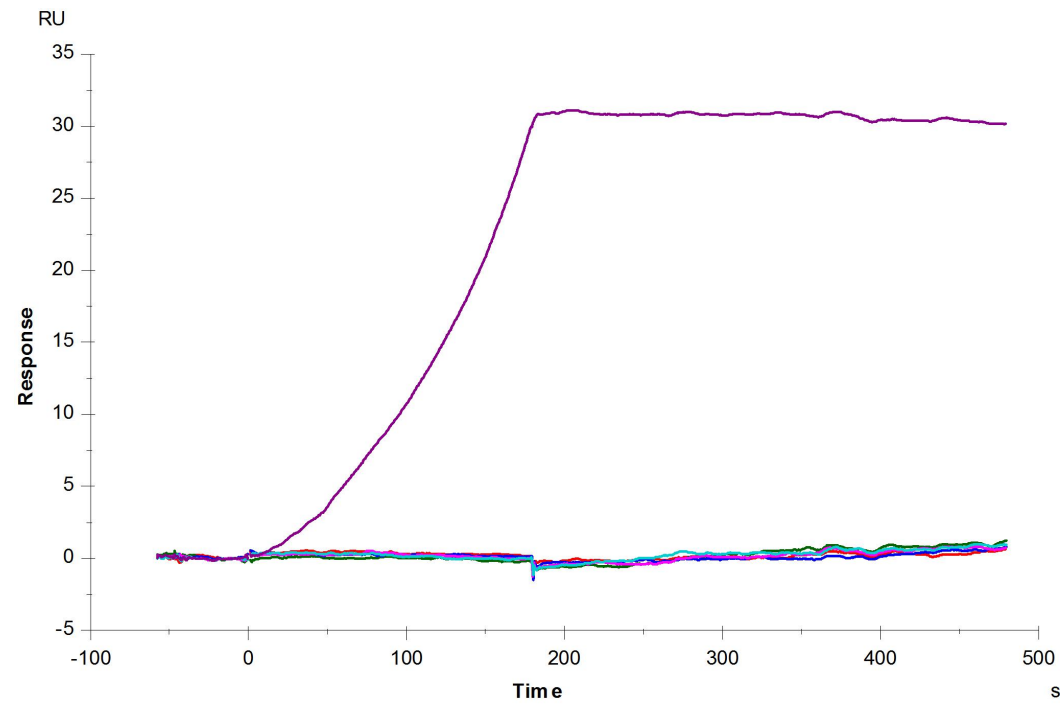

Figure 4; Binding sensorgram showing Springolin A (extracted and purified from Bacillus Cereus) binding to the 
immobilized bacterial cells. Color lines below show the no binding of culture media.

\section{Conclusions}

Preliminary data conclude that the Bacillus isolated from donkey milk secreted bacteriocin-like compound is easily extracted from the culture media. The compound is characterized and found to be Syringolin A like compound from spectral studies. Binding studies show its binding to immobilized cells.

\section{Acknowledgments}

This work was supported by the National Post Doctorate fellowship (N-PDF) SERB, DST under Grant PDF/2015/000962.

\section{Conflict of interest}

The authors have declared no conflict of interest.

\section{References}

1. Walls T, Power D, Tagg J. Bacteriocin-like inhibitory substance (BLIS) production by the normal flora of the nasopharynx: the potential to protect against otitis media? Journal of Medical Microbiology, 2003;52(9): 829-833. https://doi.org/10.1099/jmm.0.05259-0.

2. Schellenberg B, Ramel C, Dudler R. Pseudomonas syringae Virulence Factor Syringolin A Counteracts Stomatal Immunity by Proteasome Inhibition, 2010; 23(10): 1287-1293. https://doi.org/10.1094.

3. Kelly NA, Reuben BG, Rhoades J, et al. Solvent extraction of bacteriocins from model solutions and fermentation broths. Journal of Chemical Technology and Biotechnology, 2000;75(9): 777-784.

4. Burianek LL, Yousef AE. Solvent extraction of bacteriocins from liquid cultures. Lett Appl Microbiol, 2000;31(3): 193-197.

5. Pangsomboon K, Kaewnopparat S, Pitakpornpreecha T, et al. Antibacterial activity of a bacteriocin from Lactobacillus paracasei HL32 against Porphyromonas gingivalis. Arch Oral Biol, 2006;51(9):784-793. doi: 10.1016/j.archoralbio.2006.03.008.

6. El-Adawy TA. Optimum production, stability, partial purification and inhibitory spectrum of antimicrobial compounds produced by Pediococcus pentosaceus DI. Nahrung,2001; 45(2), 118-124. doi: 10.1002/1521-3803(20010401)45:2<118::AID-FOOD118>3.0.CO;2-0.

7. Tabbene O, Ben Slimene I, Bouabdallah F, et al. Production of anti-methicillin-resistant Staphylococcus activity from Bacillus subtilis sp. strain B38 newly isolated from soil. Appl Biochem Biotechnol, 2009;157(3): 407-419. doi: 10.1007/s12010-008-8277-1.

8. Baggerman G, Boonen K, Verleyen P, et al. Peptidomic analysis of the larval Drosophila melanogaster central nervous system by two-dimensional capillary liquid chromatography quadrupole time-of-flight mass spectrometry. J Mass Spectrom, 2005;40(2): 250-260. doi: 10.1002/jms.744.

9. Wu L, Hao H, Wang G. LC/MS-based tools and strategies for qualitative and quantitative analysis of herbal components in complex matrixes. Curr Drug Metab, 2012;13(9): 1251-1265.

10. Ren L, Bi K, Gong P, et al. Characterization of the in vivo and in vitro metabolic profile of PAC-1 using liquid chromatography-mass spectrometry. J Chromatogr B Analyt Technol Biomed Life Sci, 2008;876(1): 47-53. doi: 10.1016/j.jchromb.2008.10.006.

11. Ashokkumar S, Krishna RS, Pavithra V, et al. Production and antibacterial activity of bacteriocin by Lactobacillus paracasei isolated from donkey milk. Int J Curr Sci 2011; 1: 109-115.

12. Rashmi D, Srinivas Sistla, SharmilaT. Study of Antagonistic Properties of Bacteria from Cow's Milk by RealTime Surface Plasma Resonance Biosensor (BIAcore). Journal of Pharmaceutical, Chemical and Biological Sciences, 2018; 5(4): 365-370.

13. Barja JL, Lemos ML, Toranzo AE. Purification and characterization of an antibacterial substance produced by a marine Alteromonas species. Antimicrob Agents Chemother, 1989;33(10): 1674-1679.

14. Teixeira ML, Dalla Rosa A, Brandelli A. Characterization of an antimicrobial peptide produced by Bacillus subtilis subsp. spizezinii showing inhibitory activity towards Haemophilus parasuis. Microbiology, 2013;159(5), 980-988.

15. Wladyka B, Wielebska K, Wloka M, et al. Isolation, biochemical characterization, and cloning of a bacteriocin from the poultry-associated Staphylococcus aureus strain CH-91. Appl Microbiol Biotechnol,2013; 97(16): 7229-7239. doi: 10.1007/s00253-012-4578-y.

16. Laue B E, Jiang Y, Chhabra SR, et al. The biocontrol strain Pseudomonas fluorescens F113 produces the Rhizobium small bacteriocin, N-(3-hydroxy-7-cis-tetradecenoyl) homoserine lactone, via HdtS, a putative novel $\mathrm{N}$-acylhomoserine lactone synthase. Microbiology, 2000;146(10): 2469-2480.

17. McClean KH, Winson MK, Fish L, et al. Quorum sensing and Chromobacterium violaceum: exploitation of 
violacein production and inhibition for the detection of N-acylhomoserine lactones. Microbiology, 1997;143(12): 3703-3711.

18. Guiochon G, Siouffi A. Study of the Performances of Thin Layer Chromatography II. Band Broadening and Plate Height Equation. Journal of Chromatographic Science, 1978; 16(10): 470-481.

19. Sánchez L, Hedström M, Delgado M, et al. Production, purification and characterization of serraticin A, a novel cold - active antimicrobial produced by Serratia proteamaculans 136. J Appl Microbiol, 2010;109(3): 936-945.

20. Molognoni L, Daguer H, de Sá Ploêncio LA, et al. A multi-purpose tool for food inspection: Simultaneous determination of various classes of preservatives and biogenic amines in meat and fish products by LC-MS. Talanta, 2018; 178, 1053-1066. https://doi.org/10.1016/J.TALANTA.2017.08.081.

21. Rashmi D, Srinivas Sistla, SharmilaT. Study of antagonistic properties of bacteria from fermented pulses by real-time surface plasma resonance biosensor (Biacore), Asian Journal of Pharmaceutical Science \& Technology. 2018; 8(1): 10-15. 